\title{
Generation of sub-pixel-level maps for mixed pixels in hyperspectral image data
}

\author{
Prem Kumar and Somdatta Chakravortty* \\ Maulana Abul Kalam Azad University of Technology, Haringhata, Nadia 721 249, India
}

\begin{abstract}
Hyperspectral data can find wide applications in classification and mapping of pure and mixed pixels in images of different land-cover types. Hyperspectral data of high spectral resolution enhance discrimination of target objects; but the low spatial resolution poses a challenge due to creation of mixed pixels. The cost of acquiring images at high resolution from sensors is high and rarely available. With images of coarser spatial resolution, it is difficult to identify the endmembers and their locations within the mixed pixel. This study utilizes the fractional abundance values of target endmembers obtained from linear spectral unmixing in locating the sub-pixels of a mixed pixel. The study illustrates the preparation of classified maps of finer spatial resolution by locating the sub-pixels through different mapping algorithms. A comparative analysis of these mapping algorithms, viz. attraction model-based sub-pixel mapping, simulated annealing, neighbourhood connectivity, cosine similarity-based mapping and Markov random fieldbased mapping has been made and an output generated. The algorithms have been implemented on standard hyperspectral datasets of Indian Pines having 200 spectral channels, Pavia University of 103 spectral channels and Jasper Ridge of 198 spectral channels. It has been observed that simulated annealing-based mapping produces higher accuracy rate than the other algorithms, whereas in terms of execution time, attraction model takes lesser time. The accuracy has been validated with the ground reference map of available standard hyperspectral datasets on which each algorithm has been tested and analysed.
\end{abstract}

Keywords: Hyperspectral data, mapping algorithms, pure and mixed pixels, spectral channels.

THE advanced AVIRIS (airborne visible infrared imaging spectrometer) optical sensor has the ability to capture images that have a narrow spectral range and high spatial resolution. These sensors are presently used by only a few research organizations that restrict the availability of their applications to researchers working in the remotesensing field. Presently, one of the trending remote sensing technologies, viz. hyperspectral imaging, utilizes a

*For correspondence. (e-mail: csomdatta@rediffmail.com) wide range of spectral variations to differentiate various land-cover classes with the huge amount of data present in it. One of the drawbacks of the hyperspectral image is its coarser resolution, which lacks detailed classification ${ }^{1}$. Due to this mixed pixels are encountered ${ }^{2}$ (presence of many land-cover classes within a pixel). To solve the problem of mixed pixels, classification at sub-pixel level has been attempted ${ }^{3,4}$. Pure pixels have higher presence of single land-cover class, whereas mixed pixels comprises of more than one class. Sub-pixel mapping (SPM) enables to discriminate the land-cover classes and their locations within each pixel. Sub-pixel classification algorithm includes evaluation of the composition of classes in the pixel as well as their assignment to appropriate locations within the pixel.

Linear spectral unmixing model enables us to retrieve the fraction of class and assign the fractions to equivalent land-cover classes within each pixel. Based on the resolution, a zoom factor is assigned which breaks each pixel to sub-pixels with the fractional values per class. In recent decades there is increase in demand of high-resolution mapping from coarse images. As a result many algorithms have been developed and used for SPM. It has been proposed linear optimization problem can be used for SPM through spatial interpolation using an isotropic variogram $^{5}$. However, the outcomes of this process resulted in isolated sub-pixels. SPM using spatial dependency attractive model has also been proposed that generates high spatial resolution as hard classification ${ }^{6,7}$. Three other improved pixel swapping techniques are avilable ${ }^{8}$, where soft classification has been used to formulate the spatial arrangements of land-cover classes. Sub-pixel shifts algorithm is a distinct algorithm with high/low accuracy rate and low processing time ${ }^{9}$. This method was used with multiple sub-pixel shifted mapping technique. Bayesian decision principle was used for the identification of samples drawn from unknown classes, to obtain the solution to a problem where the efficiency of supervised classification gets accomplished with minimum errors in classification ${ }^{10}$. Some recent methods proposed such as super-resolution using neighbourhood regression with local structure prior describes neighbourhood relationship that combines hierarchical similarity and local structure priors ${ }^{5}$. Using clustering technique and regression method, this model produced better results in comparison to the existing methods. 
The availability of higher resolution image is not adequate; so the need of mapping a coarser image to a finer one solves the problem to classify an image at high resolution $^{11}$. The intuition is to use SPM algorithm to get higher resolution mapping using a coarse image as the scope of availability of higher resolution image is low and cost is high. This study is a comparative analysis of SPM techniques, where each technique follows a different method for mapping. The SPM algorithms implemented here are sub-pixel attraction, simulated annealing, connectivity of pixels, Markov random field (MRF) and cosine similarity-based mapping. The perimeter estimation of the connected sub-pixels is used as the cost function for retrieving the best map from the highly randomly generated map in simulated annealing. Attraction model estimates the raw attraction parameter between similar sub-pixels, and sub-pixels are arranged at a finer resolution using these parameters. Connectivity model of pixels estimates the parameters of the connected pixels at different orders of connectivity (first, second, third,...) from randomly distributed SPM, where strong connectivity between the sub-pixels for all classes results in higher stability of mapping while weak connectivity produces a random map. Markov random field estimates the conditional probability of configuration of pixels and then uses a classifier based on maximum a posteriori (MAP), which picks up the most likely super-resolution map among all possible generated sub-pixel maps. Cosine similarity estimates the cosine distance measure similarity index which is used as the cost function to find the best output from the large set of randomly distributed maps. Each algorithm has its own method to allocate sub-pixels as well as classify and produce high-resolution maps. The standard hyperspectral datasets that have been used are Indian Pines, Pavia University and Jasper Ridge, which are widely used for classification.

\section{Methodology}

The hyperspectral image with its large number of spectral channels exhibits rich data. The large number of spectral channels offer enhanced features to analyse various land covers such as water, snow, glacier, terrain, etc. more efficiently and accurately in comparison to traditional imaging systems ${ }^{12,13}$. Images are classified using several sample classes, and are detected by the presence of pure endmembers within an image that can be obtained using N-FINDR algorithm ${ }^{14}$. In this study we have extracted pure endmembers using the NFINDR algorithm and then applied linear spectral unmixing to derive fractional abundance values of sub-pixels for each land-cover class within a pixel ${ }^{15}$. The unmixing equation used is given below.

$$
Z=\sum_{i=1}^{L} a_{i} s_{i}+e=A s+e .
$$

Here $Z$ represents the fractional abundance of a given $M \times 1$ column pixel vector, $s_{i}(i=1,2,3, \ldots, L)$ the spectra of endmembers, $a$ the abundance coefficients for $L \times 1$ column vector, $e$ error due noise in the spectral band, $L$ the number of endmembers, and $s(1,2,3, \ldots, L)$ is an $M \times L$ endmember matrix of given $M$-dimensional spectral band.

In the initial classification each pixel was considered to comprise $100 \%$ of a given endmember. To extract the map for mixed pixels, which comprises of more than one class, we divided the pixels into sub-pixels so that it reflects the percentage of composition within the pixel. SPM generally includes three steps: (i) Utilization and analysis of spectral mixture models to extract soft class fraction for a given coarse resolution for the original image. (ii) Breaking down of the original pixels of the coarse image into a series of sub-pixels, assuming that one sub-pixel only contains a specific class, and to obtain the number of sub-pixels for each class. (iii) Utilization of spatial distribution characteristics of classes and other prior knowledge, in order to map the sub-pixel spatial distribution (Figure 1).

To assign the number of subpixels in a given pixel we calculate

$$
n=\text { rounding off }\left(a b d_{\mathrm{i}}\right) /(1 / N) \text {, }
$$

where $i$ is the class, $a b d_{i}$ the fractional abundance within the pixel evaluated using fully constrained linear spectral unmixing and $N$ represents the zoom factor.

In general, the SPM involves three steps: (i) Determination of fractional abundance of each pixel from the image using fully constrained linear spectral unmixing (FLSU). (ii) Breaking each pixel area into sub-pixels with some factor where sum of area of all the sub-pixel equals $s^{2}$. (iii) Allocation of sub-pixels based upon class dependency or some specific criteria.

We have implemented five sub pixel mapping algorithms to achieve our objective.

\section{Attraction model-based sub-pixel mapping}

The allocation of sub-pixels within each pixel in this approach is based upon the distance between similar classes. Euclidian distance is measured between pixels and sub-pixels for the entire image with a given window of pixel size. Pixels are divided into constituent sub-pixels of given zoom factor and abundance fraction of the pixel. The distance between similar sub-pixels of a pixel with respect to its neighbouring pixel and within the pixel is measured. In order to properly allocate sub-pixels at an appropriate location, we need to assign all sub-pixels with certain conditions and constraints. The attraction values are estimated for each sub-pixel with respect to every other sub-pixel within the window of given 

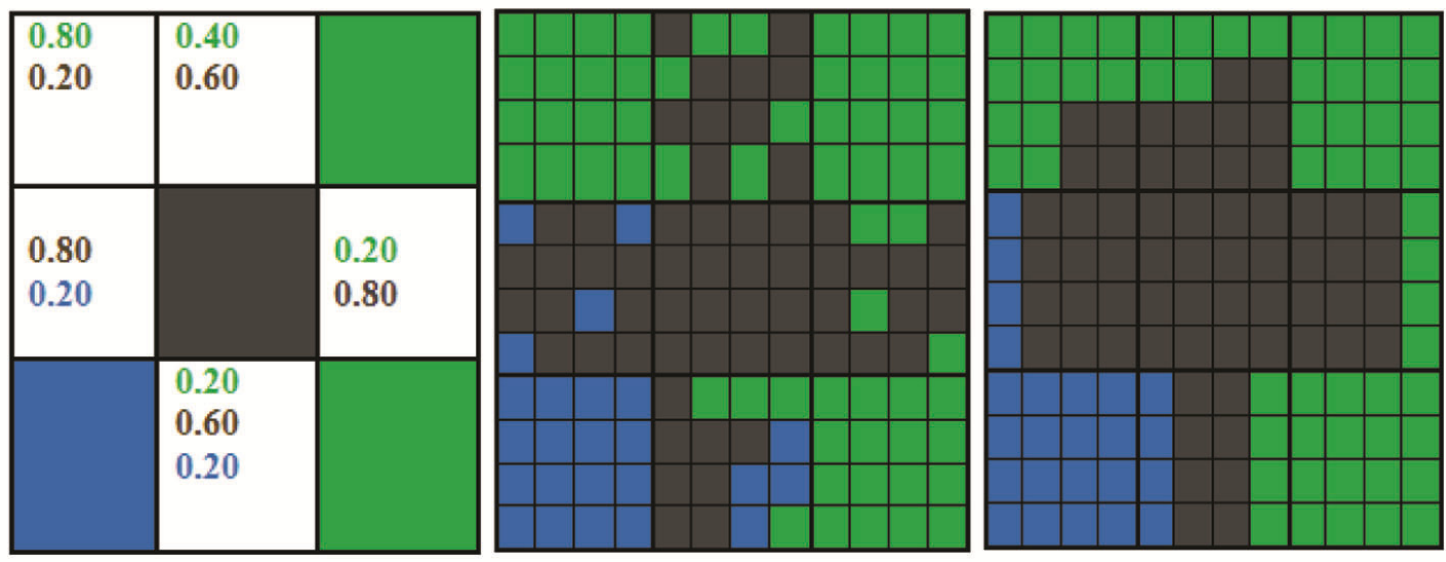

Figure 1. Steps of sub-pixel mapping.

(a)
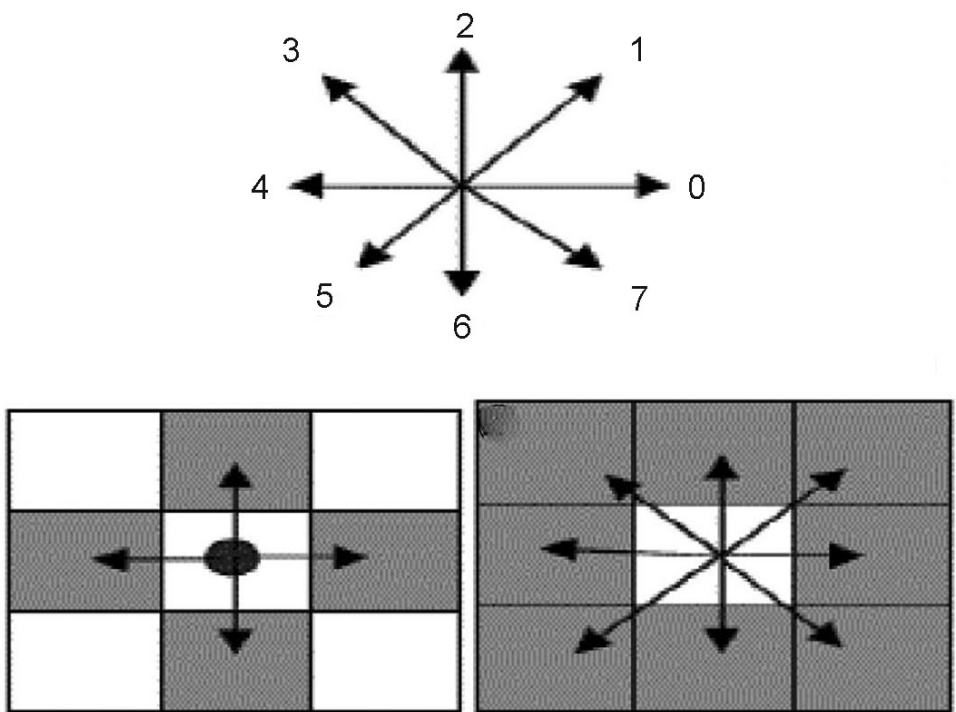

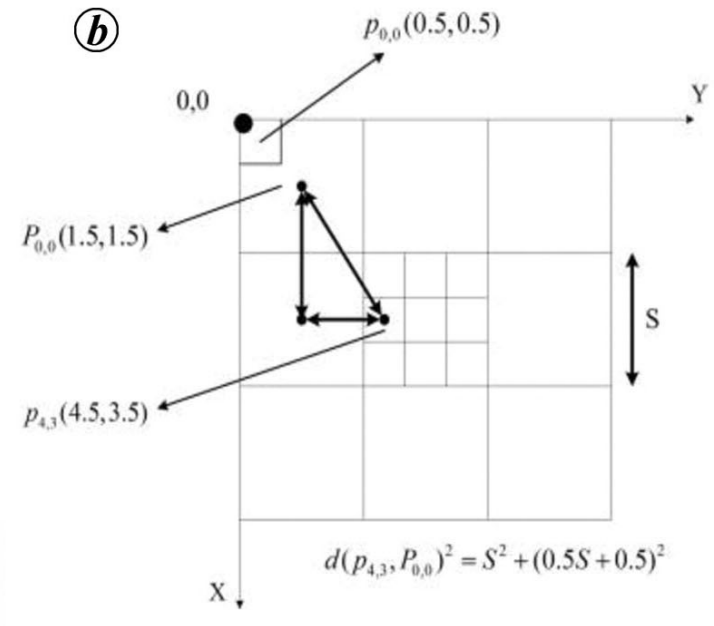

Figure 2. $\boldsymbol{a}$, Demonstration of 4, 8 neighbourhood pixels. $\boldsymbol{b}$, Distance measure between sub-pixel and neighbouring pixel.

size $^{16,17}$. Attraction values are calculated for each subpixel based upon the distance between the sub-pixels of both similar and dissimilar classes having abundance fraction of each pixel for the window frame (Figure $2 a$ and $b$ ).

The attraction value estimation uses the following equation

$$
p_{a, b}(c)=\operatorname{Avg}\left\{P_{i, j}(c) / d\left(p_{a, b}, P_{i, j}\right) \mid P_{i, j} N_{t}\left[p_{a, b}\right]\right\},
$$

where $p_{a, b}$ is the attraction value of the sub-pixel, $P_{i, j}$ the factional abundance value and $d\left(p_{a, b}, P_{i, j}\right)$ is the distance of the connected sub-pixels with its neighbourhood.

If an image has $M \times N$ dimensions, we consider a $3 \times 3$ window, i.e. nine pixels of the image. Then each pixel is broken down into constituent sub-pixels with the given zoom factor. The central pixel is used to allocate sub-pixels based on attraction values. Hence each pixel is processed by moving the given window size. The attraction values thus calculated using the above steps are assigned sub-pixels in increasing order per class, allocating each sub-pixel, and thus the finer resolution map is generated.

\section{Simulated annealing model-based sub-pixel mapping}

After extracting the fractional abundance values from linear spectral unmixing, each pixel of the image is divided into the sub-pixels with zoom factor $(z)$ assigning

CURRENT SCIENCE, VOL. 120, NO. 1, 10 JANUARY 2021 
each sub-pixel to a class using fractional value of class in the given pixel. Window of $M \times N$ pixel with a given zoom factor composed of $(M \times N) \times z^{2}$ sub-pixels is chosen and initially the sub-pixels within each pixel are randomly allocated ${ }^{2}$.

Based upon the concept that similar class sub-pixels will be closer than the dissimilar class sub-pixels, we further re-arrange the sub-pixels to allocate them. For this allocation we follow cost estimation of perimeter of sub-pixel within its neighbourhood. The cost of subpixels belonging to the same class within the window frame is calculated. To get the allocation of sub-pixels at an optimal state, random perturbation of the sub-pixel in the given window frame is carried out and the cost is calculated per class. The cost function is the area of similar sub-pixel and we select the cost where area of similar sub-pixel of a given class is minimum (eq. (3)).

$$
C=\sum_{i=1}^{I} \sum_{j=1}^{N_{i}} P_{j}
$$

Here $C$ is the cost function, $P$ the perimeter of the $i$ th class with $j$ th sub-pixel, $i$ the class and $j$ is the number of sub-pixels. If we consider a $3 \times 3$ pixel window with a sub-pixel zoom factor of 3, the sub-pixels having more than $90 \%$ of similar endmembers are pure pixels, whereas sub-pixels with endmembers less than $90 \%$ similarality are considered as mixed pixels.

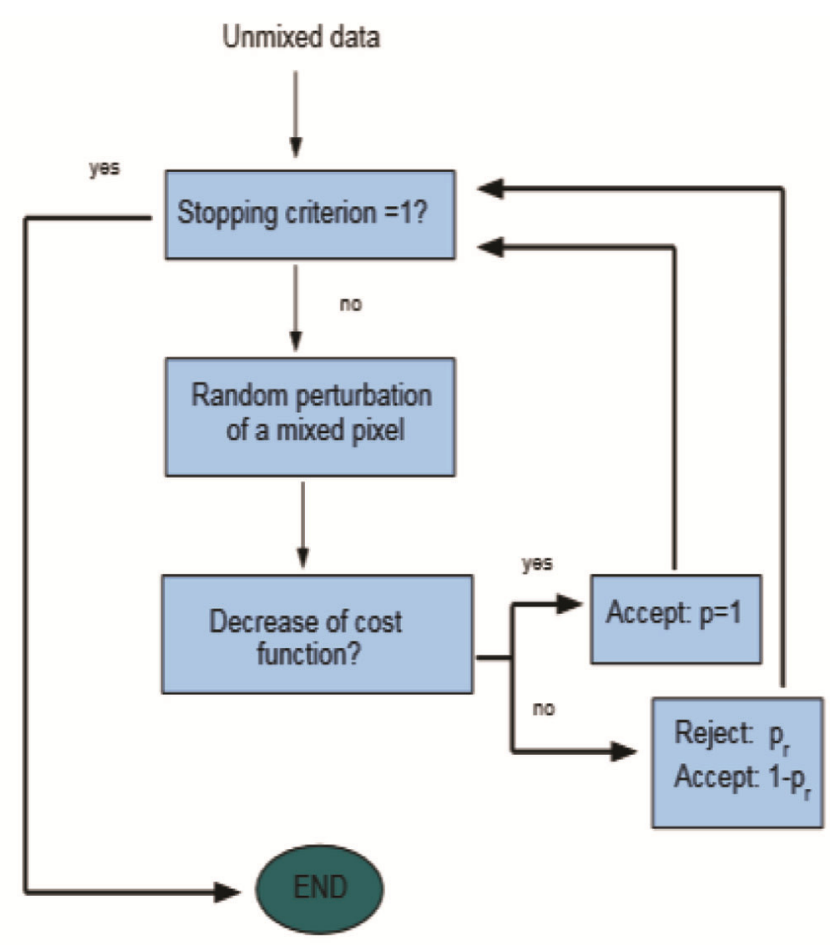

Figure 3. Steps of simulated annealing model based sub-pixel mapping.
Figure 3 underlines these steps. The approach will produce output with good allocation of sub-pixels for which cost function is to be minimized. The iteration for random perturbations is set to a maximum. At each iteration, cost of the function is measured with its previous optimal cost. If any iteration produces a state having lesser cost, the state is stored and if it gives higher cost, the iteration is continued. That is, if the cost function increases, it rejects the configuration and if cost is less the state is selected. In this study we have set counter value 100 as rejection, i.e. if continuously 100 times the function does not find the low cost preceding the iteration, it will terminate the iteration and select the last low configured state cost of the sub-pixel allocation.

Thus each pixel is processed with a window frame and output is obtained with finer resolution image.

\section{Neighbourhood connectivity-based sub-pixel mapping}

Fractional abundance of coarse image with zoom factor is used to break each pixel of the image into sub-pixels after which initially all the sub-pixels are randomly allocated. Then we assign the position of each sub-pixel based on the concept that identical class sub-pixels are mostly associated together. The allocation of sub-pixels depends on their neighbour connectivity. Basically, there are three types of connectivity of pixels: four-connected neighbours, eight-connected neighbours and $m$-connected neighbours. So for a given image, pixel to pixel connectivity can be determined using the connectivity criteria. Each pixel of an image can be classified as strongly connected pixel as well as pixels of weak connectivity. Strongly connected pixels can be easily identified by estimating the number of pixels connected to their neighbours, more the number of connected pixel, stronger is the connectivity in the neighbourhood system ${ }^{18}$.

Value assigned to a pixel at the 'centre' position is a function of its neighbourhood system and a set of window functions (eq. (4)).

$$
\begin{aligned}
p & =\left(w_{1} b+w_{2} b+w_{3} b+w_{4} b+w_{5} b+w_{6} b+w_{7} b+w_{8} b\right) \\
& =\sum w_{i} f_{i},
\end{aligned}
$$

where $w_{i}$ is the weight of the connected sub-pixel and $b$ is the sub-pixel in the neighbourhood within the window. This technique can also be used at the sub-pixel level too. For a good arrangement we initially make random allocation of the sub-pixel in a given window frame and move the window in the entire image. The weight of connectivity is determined for each class of each pixel. Then all the sub-pixels are rearranged using random allocation and weight of the new connectivity is obtained. A complete 
iteration makes the image with updated sub-pixel allocation and connectivity value of each class is calculated in this state. The process is to be repeated for a given number of iteration values and the state in which weight of connectivity is maximum; the sub-pixel are thus in the strongest state. The image generated at the strongest state produces a good arrangement of sub-pixels and a comparative analysis can be made between the coarse resolution image and newly generated high resolution image. The connectivity is assumed to be checked up to fifth order of connected sub-pixels. Increase in the order of connectivity yields more accuracy in mapping. Figure $4 a-c$ shows the order of connectivity.

The second-order connectivity is used in this study with zoom factor 3 and a $3 \times 3$ window frame.

The output is used in the analysis for accuracy with other methods of classification of finer resolution mapping.

\section{Sub-pixel mapping based on Markov random field}

The coarse spatial resolution image $Y$ with $M \times N$ pixels where each pixel has an area $a$, generates a fine resolution image $X$ having $a M \times a N$ pixels. Assume that the coarse spatial image contains $L$ number of classes of land cover that consist of mixed pixels. In the coarse spatial image each pixel may or may not be pure and can be a mixture of different classes of land cover, whereas pixels of finer spatial resolution image are pure representing a single class of land cover.

All sites (pixels) belonging to SRM are denoted by a set $T$. The coarser resolution image (hyperspectral image) has a large number of bands $(K)$. Each pixel in the SRM is considered as pure with the configuration $x(t)$ denoting a single land-cover class $(x(t) \in\{1, \ldots, L\}$. This means $L a^{2} M N$ dissimilar super-resolution maps $x(T)$ can be generated with each having different class assignments in at least one pixel.

Considering that Super Resolution Mapping (SRM) has MRF property, i.e. the conditional probability for a pixel with respect to its configuration of entire image excluding the pixel that we are considering is equivalent to the conditional probability of the pixel's configuration to its neighbouring pixels.

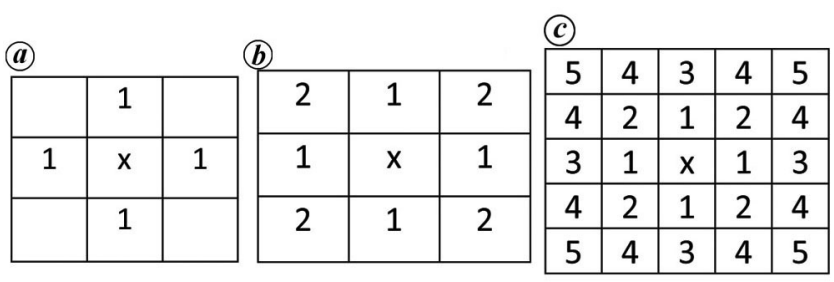

Figure 4. First (a), second (b) and fifth (c) orders of connectivity.
That can be represented as follows.

$$
\operatorname{Pr}(x(t) \mid X(T \backslash t))=\operatorname{Pr}\left(x(t) \mid X\left(N_{t}\right)\right),
$$

where $T \backslash t$ is the set of all pixels of $T$, excluding pixel $t, N_{t}$ is set of the neighbouring pixels of the given pixel $t$. That is, land cover class associated to neighbouring pixels are more likely to occur closer to the similar land-cover class pixels rather the isolated pixels. The conditional probability density function depends on the condition if a pixel has a configuration similar to its neighbouring pixels, it takes the form of Gibbs distribution as in eq. (6).

$$
\operatorname{Pr}(X)=(1 / Z) \exp \left[-\sum V_{c}(X)\right] C \zeta T
$$

where normalizing constant is denoted by $Z, C$ represents a clique, and $V_{c}(X)$ represents the Gibbs potential function. A clique is any subset of $T$ or a singleton of $T$ or whose two peculiar elements are mutually neighbours. Cliques can be four and eight neighbourhood systems. Gibbs potential function yields values depending upon the configuration of cliques and the entire SRM.

Further assume a finer spatial resolution image in which each pixel is specified with one class only, having a normal distribution. The PDF with mean vector $\mu_{i}$ and covariance matrix $\sum_{i}$ for each land-cover class is given by

$$
\begin{aligned}
& \operatorname{Pr}(z \mid x-1)=1 /(2 \pi)^{k / 2} \sqrt{\left|\operatorname{det}\left(\Sigma_{i}\right)\right|} \\
& \times \exp \left[-1 / 2\left\{\left(z-\mu_{i}\right)^{\prime} \sum_{i}^{-1}\left(z-\mu_{i}\right)\right\}\right],
\end{aligned}
$$

where the finer spatial resolution image can be represented as vector $z$ where $a \times a$ pixels of the finer resolution image represent one pixel in the coarse image. Thus the observed vector $y(s)$ of the coarser spatial resolution image having $\mathrm{PDF}$ is also assumed to be normally distributed with mean vector and a covariance matrix

$$
\mu(s)=\sum_{l=1}^{L} b_{i}(s) \mu_{i}, \text { and } \sum(s)=\sum_{l=1}^{L} b_{l}(s),
$$

where $b_{l}(s)$ is the percentage of land-cover class-1 present in $T^{s}$ such that

$$
\sum_{l=1}^{L} b_{l}(s)=1
$$

in addition, we presume that a pixel at coarse spatial resolution has a number of pure pixels at fine spatial 
resolution and the distribution of pixels is independent, since the mean vector and covariance matrix from the observed data at coarser spatial resolution are the directional sum of mean vectors and covariance matrices of the pixel at fine spatial resolution respectively.

Therefore, conditional PDF for the coarse resolution image observed can be written as

$$
\operatorname{Pr}(Y \mid X)=\prod \operatorname{Pr}(y(s) \mid b(s))
$$

where $b(s)=\left[b_{1}(s) \ldots B_{L}(s)\right]^{T}$.

The classifier based on MAP picks up the most likely SRM among all possible ones given the observed image.

The map criterion is expressed as follows

$$
X_{\mathrm{opt}}=\arg \left\{\max _{x}[\operatorname{Pr}(X \mid Y)]\right\},
$$

where $\operatorname{Pr}(\mathrm{X} \mid \mathrm{Y})$ represents the posterior probability of the SRM when a coarser spatial resolution observed image is available.

That is,

$$
X_{\mathrm{opt}}=\arg \left\{\min _{x}[E(X \mid Y)]\right\} .
$$

Since the possibility of SRM generation is too large for reducing the execution time simulated annealing (SA) algorithm might be employed to generate sequence of $\left(X_{p}\right\}, p=1,2,3, \ldots$ SA allows the best solution for eq. (5).

Overall, two major steps are involved: (i) Initialization is performed for the determination of a fairly good initial assessment of the SRM. (ii) Iteration is done for determination of the best SRM.

Here, the maximum likelihood estimation (MLE)derived probabilities are used to perform the sub-pixel classification and generate fraction images. These fraction images are further induced into a super-resolution map generator in order to get an initial resolution map at fine spatial resolution based on the following process. The pixels represented in coarse resolution image is in similar proportions to pixels corresponding to the finer resolution image ${ }^{9,19,20}$. If a fraction image of class $A$ has a value of 0.5 in pixel $s j$, there are $0.5 a^{2}$ pixels out of $a^{2}$ in the set $j$ belonging to class $\mathrm{A}$ in the SRM. A similar procedure is adopted for each fraction image and pixels in the set $j$ are then randomly labelled with all the classes. This is implied as the initial SRM. It is expected for the initial SRM to have a large amount of isolated pixels in conjunction with the neighbouring pixels belonging to different land-cover classes. For the iteration phase initial SRM is exploited as starting input for the search process. An appropriate outset is likely to result in quicker rate of convergence for the SA algorithm. Consistently, the count of isolated pixel numbers in the SRM is reduced because the contextual information present in the Gibbs potential functions VC forces the SA algorithm to iteratively generate a new SRM, which leads closer to the solutions MAP criterion which is the desired best SRM. The iteration condition set to a phase when $0.1 \%$ pixels positions are unchanged.

\section{Cosine similarity-based sub-pixel mapping}

Cosine similarity measures the similarity between two vectors of an inner product space. It is measured by the cosine of the angle between two vectors and determines whether they are pointing in roughly the same direction.

The cosine of two non-zero vectors is calculated using the Euclidean dot product formula

$$
A \bullet B=\|A\|\|B\| \cos \theta
$$

For the given two vectors of attributes $A$ and $B$, the cosine similarity $\cos (\theta)$ is represented using a dot product and magnitude as follows

$$
\text { Similarity }=\cos (\theta)=\frac{A \cdot B}{\mid A\|\| B \|}=\frac{\sum_{i=1}^{n}\left(A_{i} B_{i}\right.}{\sqrt{\sum_{i=1}^{n} A_{i}^{2} \sqrt{\sum_{i=1}^{n} B_{i}^{2}}}} .
$$

The results thus produced can be categorized as the range of similarity index from -1 to 1 , where -1 denotes lesser similarity, 0 as the same order of similarity and 1 considered as maximum similarity ${ }^{21}$.

The cosine distance measure similarity index was used as the cost function in order to select the best output from the large set of randomly generated possible outcomes from random distributed sub-pixels at finer resolution mapping.

\section{Results and discussion}

Lesser availability of higher resolution hyperspectral image has led to the implementation of various algorithms in this study to generate sub-pixel level mapped images. Application of SPM algorithms on an image of coarse spatial resolution generates a higher spatial resolution image that is then classified and compared with ground data. The accuracy level of the mapped image signifies prioritization of SPM methods for application on coarse hyperspectral images. The fractional abundance generated for each pixel using linear spectral unmixing is fed into the mapping model that produces a finer image which is compared and tested with the ground reference map of the datasets under study, namely 
Table 1. Sub-pixel mapping analysis with Indian Pines dataset (a pixel in each dataset represents 30 sq. $\mathrm{m}$ area. Each pixel in the output figures represents 10 sq. $\mathrm{m}$ area)

\begin{tabular}{|c|c|c|c|c|c|}
\hline Method & Dataset & Ground truth & Output image & $\begin{array}{c}\text { Overall accuracy } \\
(\%)\end{array}$ & $\begin{array}{c}\text { Kappa } \\
\text { coefficient }\end{array}$ \\
\hline Attraction model & & & & 86 & 0.84 \\
\hline Connected pixel classif & & & & 88 & 0.86 \\
\hline Simulated annealing & & & & 91 & 0.89 \\
\hline MRF model & & & & 87 & 0.86 \\
\hline $\begin{array}{l}\text { Cosine similarity-based } \\
\text { classification }\end{array}$ & & & & 83 & 0.82 \\
\hline
\end{tabular}

Class and legend used for Indian Pines dataset

\begin{tabular}{|c|c|c|c|c|c|}
\hline Class sample & Legend no. & Legend & Class sample & Legend no. & Legend \\
\hline Alfalfa & 1 & & Oats & 9 & \\
\hline Corn - notill & 2 & & Soybean notill & 10 & \\
\hline Corn mintill & 3 & & Soybean mintill & 11 & \\
\hline Corn & 4 & & Wheat & 12 & \\
\hline Grass pasture & 5 & & Woods & 13 & \\
\hline Grass-tree & 6 & & Buildings grass tree drives & 14 & \\
\hline Grass pasture mowed & 7 & & Stone steel towers & 15 & \\
\hline Hay-windrowed & 8 & & & & \\
\hline
\end{tabular}


RESEARCH ARTICLES

Table 2. Sub-pixel mapping analysis with Jasper Ridge dataset

\begin{tabular}{|c|c|c|c|c|}
\hline Method & Dataset & Ground truth & Output image & $\begin{array}{c}\text { Overall } \\
\text { accuracy (\%) }\end{array}$ \\
\hline Attraction model & & & & 90 \\
\hline Connected pixel classification & & & & 92 \\
\hline Simulated annealing & & & & 95 \\
\hline MRF model & & & & 91 \\
\hline Cosine similarity-based classification & & & & 92 \\
\hline
\end{tabular}

\begin{tabular}{lcccc}
\multicolumn{5}{c}{ Class and legend used for Jasper Ridge dataset } \\
\hline Class/legend & 1 & 2 & 3 & 4 \\
\hline $\begin{array}{l}\text { Class sample } \\
\text { Legend }\end{array}$ & Tree & Water & Road & Soil \\
\hline & & & & \\
\hline
\end{tabular}

Indian Pines dataset (Table 1), Pavia University (Table 2) and Jasper Ridge image (Table 3).

Pavia University dataset is the scene over Pavia, northern Italy and the study imagery has $610 \times 310$ dimensions with 103 spectral bands and nine identified endmembers. The Indian Pines dataset with $145 \times 145$ dimension and CURRENT SCIENCE, VOL. 120, NO. 1, 10 JANUARY 2021
200 spectral bands has 16 identified endmembers, of which 15 were used to classify images consisting of agriculture, forest, some built-up structures and crop. One endmember was eliminated as it had less presence and the breakdown process to constituent sub-pixels was not possible. The Jasper Ridge dataset has four endmembers 
Table 3. Sub-pixel mapping analysis with Pavia dataset

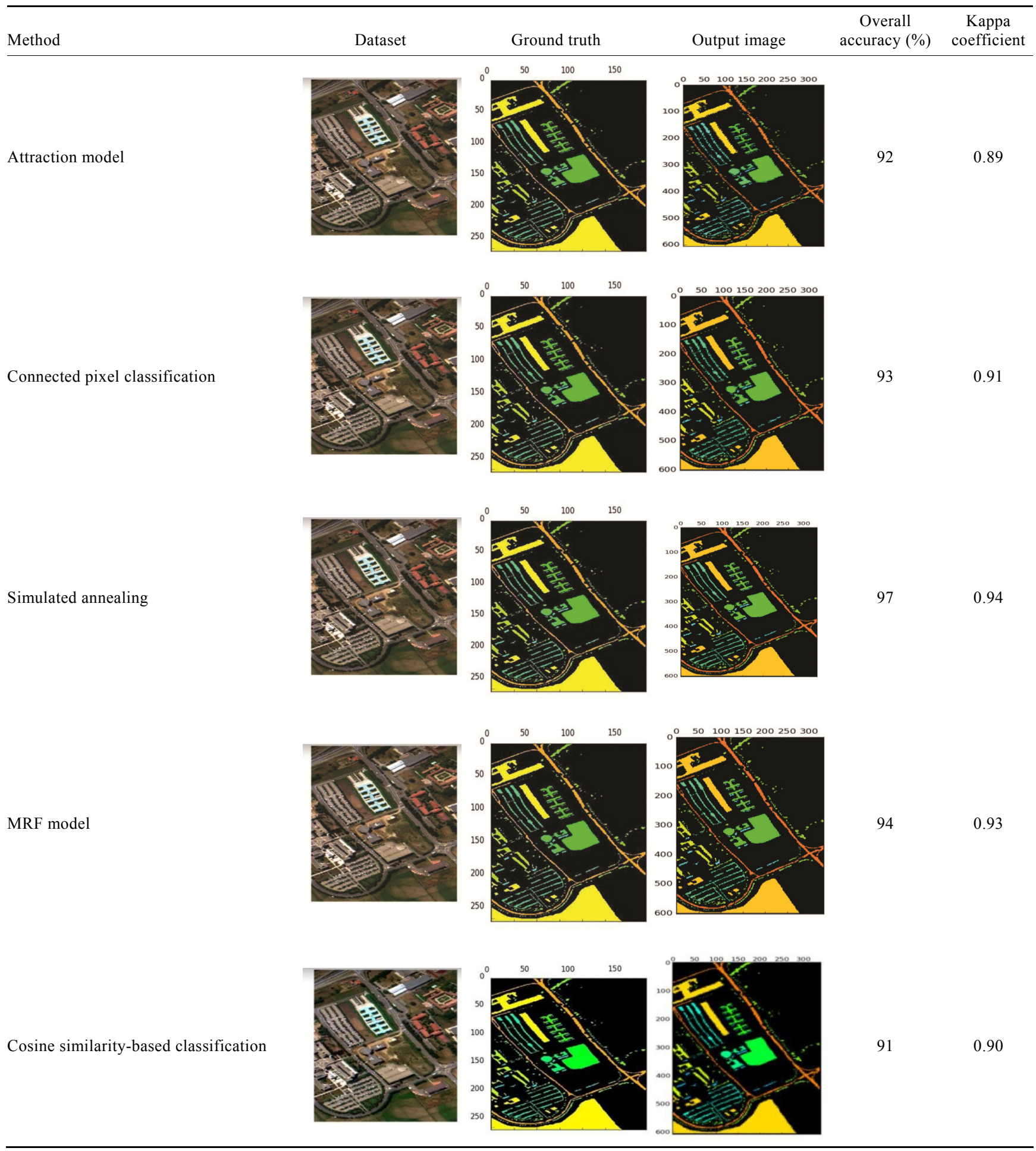

Class and legends used for Pavia dataset

\begin{tabular}{lccccccccc}
\hline Class/legend & 1 & 2 & 3 & 4 & 5 & 6 & 7 & 8 & 9 \\
\hline $\begin{array}{l}\text { Class sample } \\
\text { Legend }\end{array}$ & Alphalt & Meadows & Gravel & Tree & Painted metal sheet & Bare soil & Bitumen & Self-blocking bricks & Shadows \\
\hline
\end{tabular}


which have been used for classification with $100 \times 100$ dimension and 198 spectral bands.

All the three datasets were implemented to obtain the resultant map. Sixteen sample classes were chosen to test on the Indian Pines dataset. The dataset was downscaled and the algorithms were applied to produce a map at resolution similar to a containing ground data. Since we have downscaled the image dataset, it is easier to map the output with its ground reference. Similarly, Pavia University dataset was also compared with the available ground reference map. Jasper Ridge dataset already had fractional abundance values as ground truth and was directly considered for SPM.

The accuracy assessment signifies that SA-based mapping produces higher accuracy rate than the other two, whereas attraction model uses lesser time than the other models (Table 1-3). The cost estimation parameters for SA-based mapping depend upon the number of classes in a given image. In our test case, it was found that after setting the number of iterations to 1000 , the minimum perimeter of the connected pixel was obtained. Setting of iteration is an important factor for achieving good results. SA yields good output in less time if number of classes and image dimension are less. Attraction model is efficient for high-resolution images with greater number of classes as the model computes position in terms of attraction, which takes less computation time. MRF model enables us to map the sub-pixels, provided the images have a large number of pure pixels. Due to variation in pixel properties and abundance ratio, it is difficult to estimate the solution for which the model continues to move to infinite random maps. One of the quickest method to find the mapping of sub-pixels is the similarity index, as it takes less computational time, but one of the drawbacks is to predict the degree of accuracy level. A comparative analysis of all the four models suggests that SA is the most preferred in terms of accuracy, whereas to produce a map in lesser time, the attraction model is preferred. It is also observed that connectivity-based maps have greater accuracy than those of the attraction model. The MRF produces quite high accuracy than connectivity model but it is lower than that of SA model.

\section{Conclusion}

This study compares four SPM algorithms for enhancing spatial resolution of coarse hyperspectral images. We utilized the results of linear spectral unmixing as input for determination of spatial distribution of sub-pixels for the datasets of Pavia, Jasper and Indian Pines within a mixed pixel area. The sub-pixel locations were determined using SA algorithm, attraction model, connectivity-based model, MRF model and cosine similarity-based model. We have successfully reproduced an accurate classified map of finer spatial resolution. The SA algorithm is found to be the most accurate when matched with the ground truth data available with standard datasets. The attraction model takes the lowest execution time to generate maps. In the algorithms implemented, two key factors that affect the classification precision of pixels and sub-pixels are the threshold used to govern the purity of a pixel and the number of endmembers measured as input for classification. If more number of endmembers are measured, it may so happen that a specific endmember may not essentially be spatially close to the selected pixel. Hence, including such endmembers for sub-pixel classification would introduce useless information, thus affecting classification precision. Furthermore, increasing the zoom factor leads to an increase in possible sub-pixel mixtures, which would in turn escalate the computational weight exponentially.

The methods discussed here help overcome the drawbacks of low spatial resolution of hyperspectral data by enhancing spatial details using the implemented SPM algorithms.

1. Atkinson, P. M., Mapping sub-pixel boundaries from remotely sensed images. In Innovations in GIS4 (ed. Kemp, Z.), Taylor and Francis, Bristol, PA, 1997, pp. 166-169.

2. Villa, A. Chanussot, J., Benediktsson, J. A. and Jutten, C., Spectral unmixing for the classification of hyperspectral images at a finer spatial resolution. IEEE J. Sel. Top. Signal Process., 2011, 5(3), 521-533.

3. Choodarathnakar, A. L., Kumar, T. A., Koliwad, S. and Patil, C. G., Mixed pixels: a challenge in remote sensing data classification for improving performance. Int. J. Adv. Res. Comput. Eng. Technol., 2012, 1(9), 261-271.

4. Ling, F., Du, Y., Xiao, F., Xue, H. and Wu, S., Super-resolution land-cover mapping using multiple subpixel shifted remotely sensed images. Int. J. Remote Sensing, 2010, 31(19), 50235040 .

5. Verhoeye, J. and Wulf, R., Land cover mapping at sub-pixel scales using linear optimization techniques. Remote Sensing Environ., 2002, 79, 96-104; 10.1016/S0034-4257(01)00242-5.

6. Suresh, M. and Jain, K., Semantic driven automated image processing using the concept of colorimetry. J. Proc. Comput. Sci., 2015, 129(1), 453-460.

7. Suresh, M. and Jain, K., Subpixel level arrangement of spatial dependences to improve classification accuracy. In IEEE International Conference on Advances in Computing, Communication and Informatics (ICACCI), Cochin, 2015, pp. 799-786, 978-14799-8792-4/15/\$31.00@2015.

8. Niroumand, M. J., Safadarinczhad, A. R., Shaebi, M. R., A novel approach to super resolution mapping of multispectral imagery based on pixel swapping technique. Ann. Photogramm. Remote Sensing, 2012, 7, 159-164.

9. Li, J., Bioucas-Dias, J. M. and Plaza, A., Spectral-spatial hyperspectral image segmentation using subspace multinomial logistic regression and Markov random fields. IEEE Trans. Geosci. Remote Sensing, 2012, 50(3), 809-823.

10. Mantero, P., Moser, G. and Serpico, S. B., Partially supervised classification of remote sensing images through SVM-based probability density estimation. IEEE Trans. Geosci. Remote Sensing, 2005, 43(3), 559-570.

11. Sung, P., Min, P. and Moon, K., Super-resolution image reconstruction: a technical overview. IEEE Signal Process. Mag., 2003, 20, 21-36; 10.1109/MSP.2003.1203207. 
12. Plaza, A. et al., Recent advances in techniques for hyperspectral image processing. Remote Sensing Environ., 2009, 113, S110 $\mathrm{S} 122$.

13. Shafri, H. Z., Taherzadeh, E., Mansor, S. and Ashurov R., Hyperspectral remote sensing of urban areas: an overview of techniques and applications. Res. J. Appl. Sci. Eng. Technol., 2012, 4(11), 1557-1565.

14. Xiong, W., Wu, C.-C., Chang, C.-I., Kapalkis, K. and Chen, H. M., Fast algorithms to implement N-FINDR for hyperspectralendmember extraction. IEEE J. Sel. Top. Appl. Earth Obs. Remote Sensing, 2011, 4, 545-564.

15. Broadwater, J., Chellappa, R., Banerjee, A. and Burlina, P., Kernel fully constrained least squares abundance estimates. In Proceedings of IEEE International Geoscience and Remote Sensing Symposium, Barcelona, Spain, 2007, pp. 4041-4044.

16. Tong, X., Zhang, X., Shan, J., Xie, H. and Liu, M., Attractionrepulsion model-based subpixel mapping of multi-hyperspectral imagery. IEEE Trans. Geosci. Remote Sensing, 2013, 51(5), 27992814; doi:10.1109/TGRS.2012.2218612.

17. Lizhen, L., Huang, Y., Liping, D. and Danwei, H., A new spatial attraction model for improving subpixel land cover classification.
Remote Sensing Environ., 2005, 96(3-4), 302-314; doi:10.3390/ rs9040360.

18. Bin, A., Xiaoping, L., Guohua, H. and Xia, L., Improved sub-pixel mapping method coupling spatial dependence with directivity and connectivity. IEEE J. Sel. Topics Appl. Earth Obs. Remote Sensing, 2014, 7(12), 4887-4896; doi:10.1109/JSTARS.2014.2313978.

19. Kasetkasem, T., Arora, M. K. and Varshney, P. K., Superresolution land cover mapping using a Markov random field based approach, 2005; https://doi.org/10.1016/j.rse.2005.02.006.

20. Bhatt, M. and Desai, U. B., Robust image restoration algorithm using markov random field model. Graph. Models Image Process., 1994, 56(1), 61-74.

21. Hirai, A. and Tonooka, H., Mineral discrimination by combination of multispectral image and surrounding hyperspectral image. $J$. Appl. Remote Sensing, 2019, 13(2), 024517; https://doi.org/ 10.1117/1.JRS.13.024517.

Received 8 March 2020; rerevised accepted 29 July 2020

doi: $10.18520 / \mathrm{cs} / \mathrm{v} 120 / \mathrm{i} 1 / 166-176$ 